\title{
TU/e EmonOWEN

\section{Investigations of Stark effects in xenon Rydberg states by laser-induced fluorescence-dip spectroscopy}

\section{Citation for published version (APA):}

Wagenaars, E., Kroesen, G. M. W., \& Bowden, M. D. (2006). Investigations of Stark effects in xenon Rydberg states by laser-induced fluorescence-dip spectroscopy. Physical Review A : Atomic, Molecular and Optical Physics, 74(3), 033409-1/7. [033409]. https://doi.org/10.1103/PhysRevA.74.033409

DOI:

10.1103/PhysRevA.74.033409

Document status and date:

Published: 01/01/2006

\section{Document Version:}

Publisher's PDF, also known as Version of Record (includes final page, issue and volume numbers)

\section{Please check the document version of this publication:}

- A submitted manuscript is the version of the article upon submission and before peer-review. There can be important differences between the submitted version and the official published version of record. People interested in the research are advised to contact the author for the final version of the publication, or visit the $\mathrm{DOI}$ to the publisher's website.

- The final author version and the galley proof are versions of the publication after peer review.

- The final published version features the final layout of the paper including the volume, issue and page numbers.

Link to publication

\section{General rights}

Copyright and moral rights for the publications made accessible in the public portal are retained by the authors and/or other copyright owners and it is a condition of accessing publications that users recognise and abide by the legal requirements associated with these rights.

- Users may download and print one copy of any publication from the public portal for the purpose of private study or research.

- You may not further distribute the material or use it for any profit-making activity or commercial gain

- You may freely distribute the URL identifying the publication in the public portal.

If the publication is distributed under the terms of Article 25fa of the Dutch Copyright Act, indicated by the "Taverne" license above, please follow below link for the End User Agreement:

www.tue.nl/taverne

Take down policy

If you believe that this document breaches copyright please contact us at:

openaccess@tue.nl

providing details and we will investigate your claim. 


\title{
Investigations of Stark effects in xenon Rydberg states by laser-induced fluorescence-dip spectroscopy
}

\author{
E. Wagenaars and G. M. W. Kroesen* \\ Department of Applied Physics, Eindhoven University of Technology, P.O. Box 513, 5600 MB Eindhoven, The Netherlands \\ M. D. Bowden \\ Department of Physics and Astronomy, The Open University, Milton Keynes, MK7 6AA, United Kingdom
}

(Received 12 June 2006; published 19 September 2006)

\begin{abstract}
Stark effects in Rydberg states of xenon atoms were investigated both experimentally and theoretically. The experimental part consisted of laser-induced fluorescence-dip spectroscopy. Using a $(2+1)$-photon excitation scheme, xenon atoms were excited from the ground state to high-lying Rydberg $n s$ and $n d$ levels. Measurements were made in a controllable electric field environment, produced by applying a pulsed voltage to two parallel metal electrodes. For energy levels with principal quantum numbers ranging from 12 to 18 , Stark shifts of up to $4.8 \mathrm{~cm}^{-1}$ were observed for electric fields ranging from 0 to $4000 \mathrm{~V} / \mathrm{cm}$. Additionally, mixing of energy levels in high electric fields was measured for $n d$ levels. The experimental results were compared to a theoretical calculation based on solving the Schrödinger equation for a perturbed Hamiltonian. The calculation method proved to be very accurate for predicting Stark effects in Rydberg $n d$ levels, while for $n s$ levels the agreement was only moderate, probably due to deviations from the assumption of a hydrogenlike atom that is used in the calculation. Finally, the feasibility of using measurements of Stark shifts of Rydberg levels as a diagnostic for electric fields in low-pressure discharges was discussed.
\end{abstract}

DOI: 10.1103/PhysRevA.74.033409

PACS number(s): 32.60. $+\mathrm{i}, 52.70 .-\mathrm{m}$, 52.38.Dx

\section{INTRODUCTION}

The dc Stark effect is characterized by shifts and splitting of energy levels of atoms or molecules under the influence of external electric fields. Especially for highly excited Rydberg states, the Stark effect can be very pronounced, with shifts of energy levels much larger than the energy separation between levels. Over the last few decades, the Stark effect in Rydberg states has been studied extensively, both experimentally and theoretically. The first investigations studied alkalimetal atoms [1-4], while later also noble gases, including xenon, krypton, and argon, were investigated [5-8].

In gas discharge physics, the electric field distribution is an important quantity. In low-pressure discharges, it drives many fundamental processes and is closely connected to other plasma parameters such as charge densities and fluxes. Additionally, the electric field is often used as input for plasma modeling. Therefore, measurements of electric field distributions in plasmas are desirable.

Detection of Stark effects in atoms and molecules by laser spectroscopy can be used as a diagnostic for electric field strengths in plasmas. This technique was first demonstrated in $\mathrm{BCl}_{3}[9]$ and helium discharges $[10,11]$. Later, measurements in hydrogen and argon plasmas were performed $[12,13]$.

In recent years, Czarnetzki et al. [14] introduced a new method for the detection of Stark effects based on laserinduced fluorescence-dip (LIF-dip) spectroscopy. With this double-resonance technique the sensitivity of the measurements in a hydrogen discharge could be improved by an order of magnitude compared to conventional LIF methods.

*Electronic address: g.m.w.kroesen@tue.nl
Recently, the use of the LIF-dip technique has been extended to argon discharges $[15,16]$.

In the research described in this paper we investigated Stark effects in xenon atoms by LIF-dip spectroscopy. The goal of these investigations was twofold. First, we characterized the shifts of bound Rydberg $n s$ and $n d$ levels in externally applied electric fields for principal quantum numbers ranging from 12 to 18 . The experimental Stark spectra were compared with a theoretical calculation based on solving the Schrödinger equation for a perturbed Hamiltonian. Similar investigations, using a different excitation scheme, have been performed for autoionizing $n s$ and $n d$ Rydberg levels in xenon by Ernst et al. [17].

Second, we investigated the feasibility of using LIF-dip Stark shift measurements as an electric field diagnostic in low-pressure gas discharges. Our excitation scheme uses the ground state of the xenon atoms as the lower level, which makes it possible to measure electric fields not only in plasmas, but also in neutral gas. This is a unique feature for noble gas electric field diagnostics, making investigations of discharges with low degrees of ionization, such as plasma breakdown, possible. Additionally, electric fields in discharges in other gases than xenon can be performed by adding xenon to the discharge as a trace species.

The structure of the paper is as follows. Section II contains experimental investigations, presenting both the experimental arrangement and the results. In Sec. III a theoretical calculation method is described and the calculation results are compared with the experiments. In Sec. IV we investigate the feasibility of using our experimental technique as an electric field diagnostic in low-pressure plasmas. Section V contains the conclusions and a short outlook. 


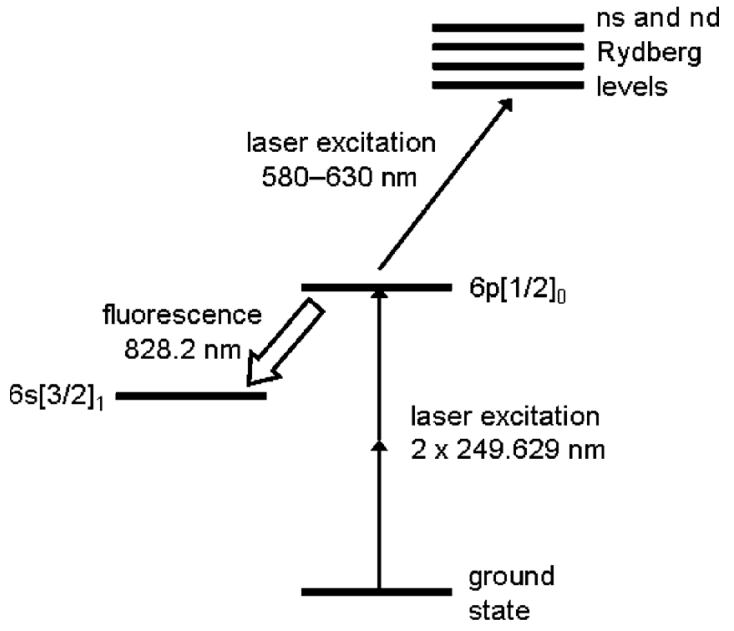

FIG. 1. Excitation scheme for LIF-dip spectroscopy in xenon.

\section{EXPERIMENTAL INVESTIGATIONS}

In this section we present our experimental investigations on Stark effects of xenon $n s$ and $n d$ levels. First, the experimental arrangement is described in Sec. II A. Next, the results of the measurements are presented in Sec. II B.

\section{A. Experimental arrangement}

The experimental arrangement consisted of a laser system for the excitation of xenon atoms to Rydberg levels, an electrode arrangement for the application of electric fields, and a detection system to monitor fluorescence light.

Stark effects in xenon were measured by probing highlying Rydberg states by LIF-dip spectroscopy. We used a $(2+1)$-photon excitation scheme, shown in Fig. 1, which was similar to that used by Ebata et al. [18] for investigations in NO and Czarnetzki et al. [14] for atomic hydrogen. First, ground-state xenon atoms were excited to the $6 p[1 / 2]_{0}$ level in a two-photon transition at $249.629 \mathrm{~nm}$. Subsequently, fluorescence light with a wavelength of $828.2 \mathrm{~nm}$ could be observed as a result of decay to the $6 s[3 / 2]_{1}$ state. A second laser was tuned to probe the transition between the intermediate $6 p[1 / 2]_{0}$ level and high-lying Rydberg $n s$ and $n d$ states. A resonance for such a transition was detected as a decrease of the fluorescence intensity, because part of the $6 p[1 / 2]_{0}$ level population was transferred to the Rydberg state. Therefore, when scanning the second laser and monitoring the fluorescence intensity, a transition to a Rydberg state was detected as a dip in the spectrum.

The two lasers necessary for the excitation were both tunable dye lasers, pumped by the same pulsed neodymiumdoped yttrium aluminum garnet (Nd:YAG) laser. The first dye laser was pumped by the third harmonic of the Nd:YAG laser and operated at $499.258 \mathrm{~nm}$. This light was subsequently frequency doubled by a beta barium borate (BBO) crystal to create $249.629 \mathrm{~nm}$ photons for the two-photon transition. The second dye laser was pumped by the second harmonic output of the same Nd:YAG laser and operated at wavelengths between 580 and $630 \mathrm{~nm}$.

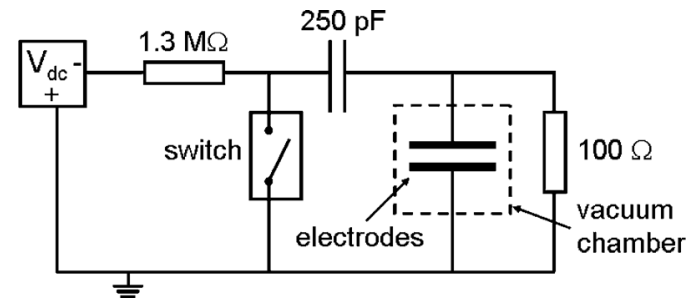

FIG. 2. Schematic diagram of the electrical arrangement for creating short voltage pulses. The high-voltage transistor switch (Behlke Electronic $\mathrm{GmbH}$ ) could be triggered externally for synchronization purposes. The electrodes were mounted inside a vacuum chamber, creating a gap of $3 \mathrm{~mm}$.

Both beams were sent to a vacuum chamber containing an electrode arrangement. The two beams were parallel to the electrode surfaces and crossed each other in between the electrodes. The energies of both lasers were reduced to a few hundred microjoules to avoid initiating a discharge and disturbing the applied electric field.

The fluorescence light was detected perpendicular to the laser beams by an intensified charge-coupled device (ICCD) camera. A lens system and an interference filter imaged the fluorescence light onto the ICCD camera, detecting only $828.2 \mathrm{~nm}$ light.

We applied a pulsed electric field to parallel plate electrodes, separated by $3 \mathrm{~mm}$, in xenon gas at $50 \mathrm{~Pa}$. The field was pulsed to prevent the formation of a discharge for voltages above the breakdown voltage of the system. Using the electrical scheme shown in Fig. 2, we could apply voltage pulses with a full width at half maximum of about $50 \mathrm{~ns}$ and an amplitude of up to $1.5 \mathrm{kV}$. In this way, the electric field in the gap was controllable and uniform.

Finally, the lasers, the ICCD camera and the voltage pulse were synchronized with a delay generator.

\section{B. Experimental results}

\section{Stark effects for nd levels}

We investigated Stark effects for $n d[3 / 2]_{1}$ levels with principal quantum numbers $n=12-15$ and $n=18$. Figure 3 shows fluorescence-dip spectra for the transition to $15 d[3 / 2]_{1}$ in different electric fields. It is clear that the dip at $586.51 \mathrm{~nm}$, corresponding to the $15 d[3 / 2]_{1}$ level, moves to longer wavelengths for increasing electric fields. Additionally, for an electric field of $2100 \mathrm{~V} / \mathrm{cm}$, we observe extra dips at wavelengths 586.40 and $586.28 \mathrm{~nm}$. These dips correspond to transitions to $13 f$ levels which are forbidden without an electric field, but become allowed when they are mixed with other levels in high electric fields.

The shifts of $n d[3 / 2]_{1}$ levels in electric field for principal quantum numbers $n=12-15$, and $n=18$ are shown in Fig. 4. For applied electric fields between 0 and $4000 \mathrm{~V} / \mathrm{cm}$, we measured Stark shifts of up to $4.8 \mathrm{~cm}^{-1}$. The measurements show that the observed shift is higher for larger principal quantum numbers, as expected.

\section{Stark effects for ns levels}

The shifts of $n s[3 / 2]_{1}$ levels in electric fields for principal quantum numbers $n=14-16$ are shown in Fig. 5. Applying 


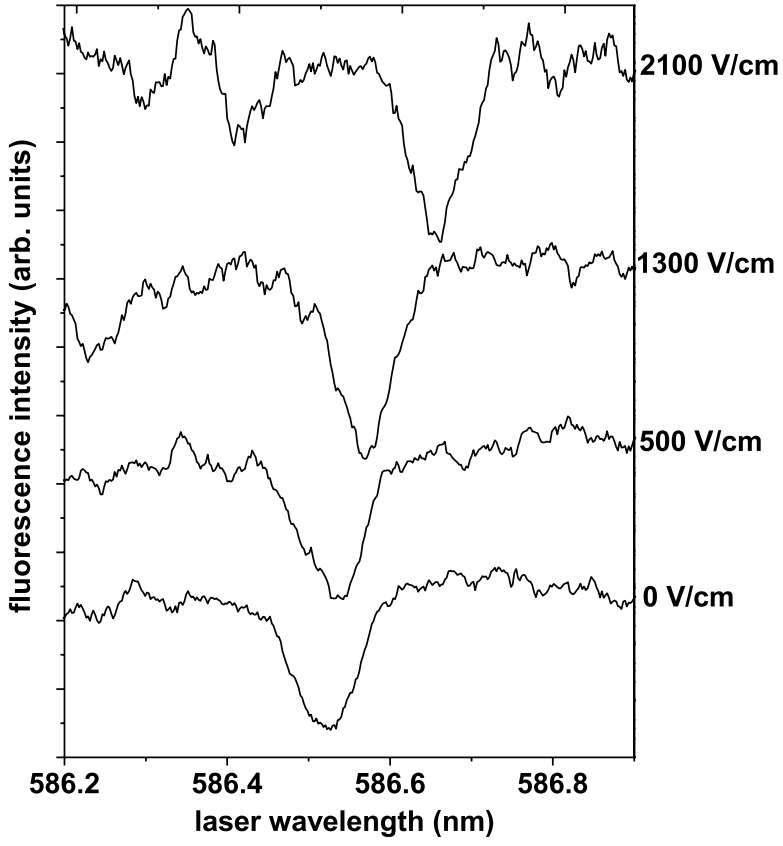

FIG. 3. Fluorescence-dip spectra showing the transition to the $15 d[3 / 2]_{1}$ state in different electric fields. A ten-point adjacent averaging of the data and an offset in the $y$ scale of the figure are applied to clarify and separate the spectra.

electric fields in the range of 0 to $4000 \mathrm{~V} / \mathrm{cm}$ resulted in Stark shifts of the $n s$ levels with a maximum of $0.6 \mathrm{~cm}^{-1}$.

When comparing these results for $n s$ levels with the Stark shifts of the $n d$ levels with similar energies (Fig. 4), it is clear that the shifts of the $n s$ levels were much smaller.

\section{THEORETICAL CALCULATIONS}

In this section a theoretical calculation of Stark effects is discussed. The calculation method and procedure are described in Sec. III A. Some representative examples of calculated results are presented in Sec. III B. Finally, in Sec. III C the calculation results are compared with the experimental results from Sec. II.

\section{A. Calculation method and procedure}

A calculation method for describing Stark structures of Rydberg states in alkali-metal atoms was introduced by Zimmerman et al. [3]. This calculation method was extended by Kelleher and Saloman to describe atoms with anisotropic cores such as barium [4]. In recent years, the method, in slightly modified form, has also been applied to noble gases such as xenon [6], argon [8,19], and krypton [20]. In these investigations the calculation of the radial matrix elements was simplified by using tables of calculations by Edmonds $e t$ al. [21] instead of calculations in the Bates-Damgaard approximation. We will also use this modified Zimmerman method for our calculations. The theoretical calculation method has been extensively described in the references mentioned above. Therefore, we will only briefly point out the main features. (i) For Rydberg levels of noble gas atoms, the $j K$-coupling scheme is most suitable [22]. In this scheme, the orbital angular momentum of the Rydberg electron $l$ is strongly coupled to the total angular momentum of the ionic core $j_{c}$. The resultant momentum $K$ is coupled to the spin of the Rydberg electron $s$, giving the total angular momentum of the atom $J$. Energy levels can now be described by wave functions of the form $\varphi=\left|n l[K]_{J}\right\rangle$, where $n$ is the principal quantum number.

(ii) The energies of Rydberg levels in electric fields can be found by numerically solving the Schrödinger equation for an atom interacting with an electric field $\mathbf{F}$ :

$$
H \psi=E \psi,
$$

with the total Hamiltonian $H$ described by

$$
H=H_{0}+e z F .
$$

Here, $H_{0}$ represents the Hamiltonian of the atom in zero field and $e z F$ the dipole interaction of the atom with the applied electric field. The axis $z$ is chosen to be parallel to the electric field vector.

(iii) At the start of the calculation procedure, the diagonal matrix $H_{0}$, of which the elements are the zero-field energies of the Rydberg levels, is constructed. Not only are the Rydberg levels involved in the laser excitation included, but also neighboring Rydberg states, because of interactions with these levels. Most of these data can be found in literature [23], but the energies of some levels had to be calculated using the method of Kelleher and Saloman [4].

(iv) The off-diagonal elements resulting from the Stark Hamiltonian, $H_{\text {Stark }}=e z F$, are calculated for each value of the electric field. The radial matrix elements, are calculated in the Coulomb approximation, using tables presented by Edmonds et al. [21]. As mentioned in Ref. [21], these tables are in agreement with the methods used by Zimmerman et al. [3] and Kelleher and Saloman [4].

(v) The total matrix $H=H_{0}+H_{\text {Stark }}$ is numerically diagonalized.

(vi) The energies of the Rydberg levels in electric fields correspond to the eigenvalues of the total Hamiltonian $H$. The transition intensities can be calculated using the eigenfunctions of $H$.

The main approximation in this calculation procedure is that the xenon Rydberg atom is a hydrogenlike atom. This assumes nonpenetrating electron orbits, which is valid for large values of $l$, but is a less appropriate approximation for small $l$ values.

It should be noted that although this calculation method involves parameters such as quantum defects and zero-field energies, we did not use adjustable parameters to fit the theory to experimental results.

\section{B. Calculation results}

As an example of the results of the calculation, a Stark map of energy levels around $15 d[3 / 2]_{1}$ is presented in Fig. 6, showing the positions of the energy levels as a function of electric field. An excitation spectrum can be calculated from the Stark maps using the eigenfunctions of the Hamiltonian 

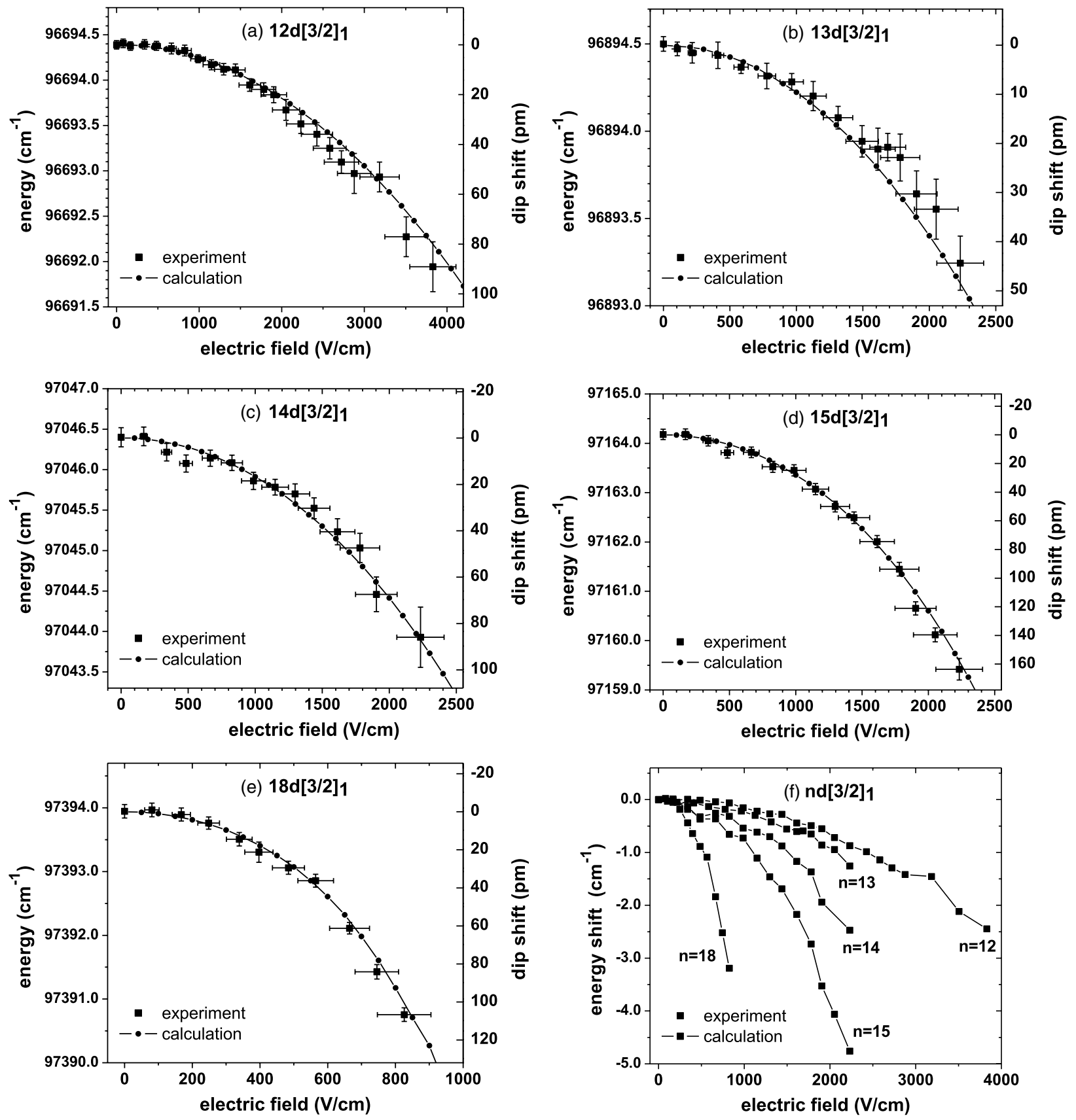

FIG. 4. Shifts of $n d[3 / 2]_{1}$ levels as a function of electric field for principal quantum numbers $n=12$ (a), 13 (b), 14 (c), 15 (d), and 18 (e). The squares ( $\square$ ) denote the experimental results, the dots with lines (-•-) represent the results of a theoretical calculation (described in Sec. III). (f) gives an overview of the shifts for all measured $n d[3 / 2]_{1}$ levels.

$H$. Without an electric field, the selection rules of $j K$ coupling only allow certain transitions. Therefore, the calculated excitation spectrum will not show transitions to all levels presented in Fig. 6, but only the transitions allowed by the selection rules. An example of a calculated spectrum for the $15 d[3 / 2]_{1}$ state in different electric fields is shown in Fig. 7. The zero-field spectrum shows only the allowed transition to the $15 d[3 / 2]_{1}$ state, the other allowed transitions $15 d[1 / 2]_{1}$ and $17 s[3 / 2]_{1}$ are outside the plotted spectral range. It is clear that the $15 d[3 / 2]_{1}$ level shifts to longer wavelengths in moderate electric fields. Additionally, in high electric fields, extra transitions which are forbidden in zero field, become allowed.

\section{Comparison of theoretical and experimental results}

From the theoretical spectra, such as shown in Fig. 7, the shifts of the $n d$ and $n s$ levels for different electric fields were determined. In Figs. 4 and 5, these calculated shifts are compared to the experimental shifts. It can be seen that the calculated results matched the experiments very well for the $n d$ levels, while for the $n s$ levels the agreement was only moderate. 

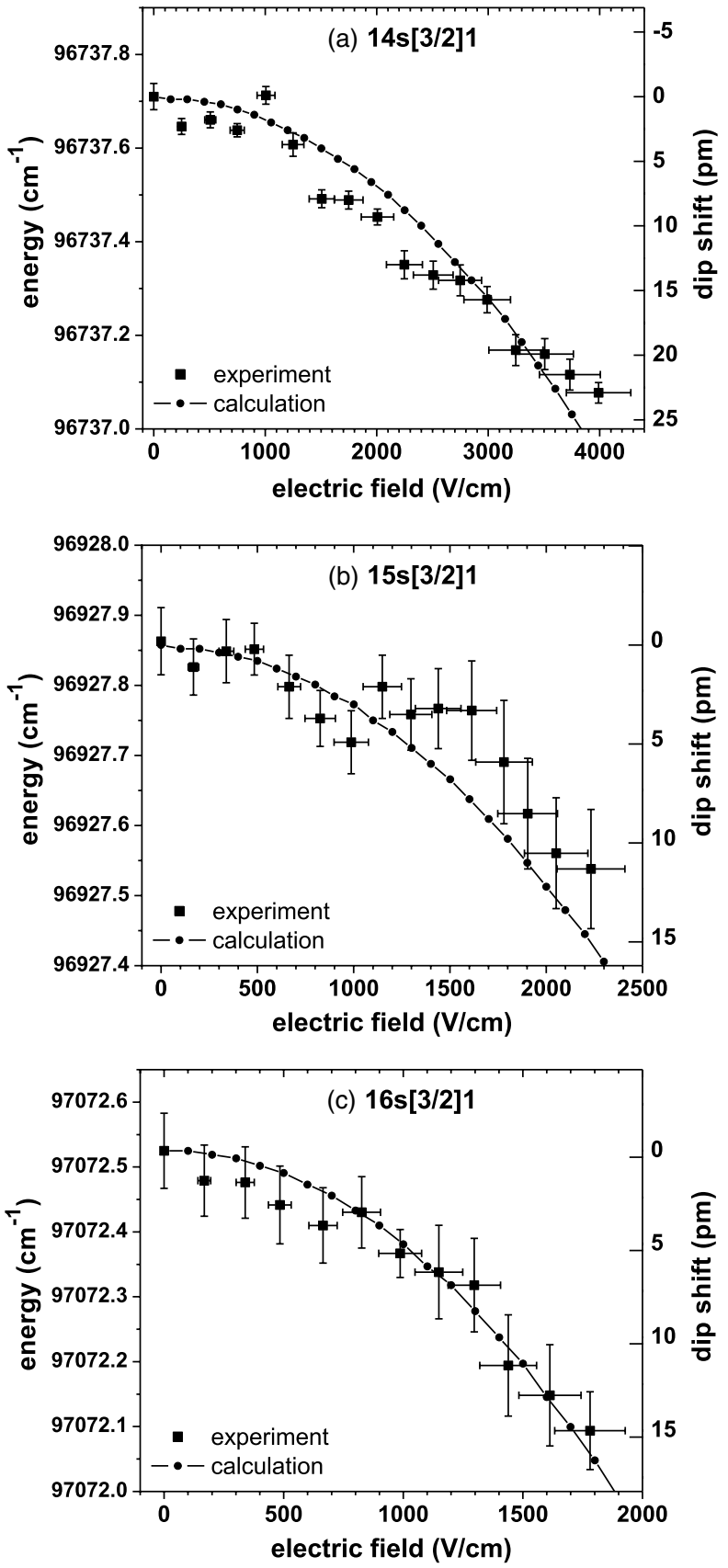

FIG. 5. Shifts of $n s[3 / 2]_{1}$ levels as a function of electric field for principal quantum numbers $n=14$ (a), 15 (b), and 16 (c). The squares $(\boldsymbol{\square})$ denote the experimental results, the dots with lines (-•-) represent the results of a theoretical calculation (described in Sec. III).

These comparisons show that the relatively simple calculation procedure can very accurately describe Stark effects of $n d$ levels in xenon Rydberg atoms. The assumption of a hydrogenlike atom with a Coulomb potential and nonpenetrating orbits appears to be valid for $n d$ levels. However, for the $n s$ levels, the calculation gives less accurate quantitative results, probably because the assumption of nonpenetrating electron orbits is not valid for these levels.

In both the experiments and the calculations, extra transitions become allowed for high applied electric fields. From

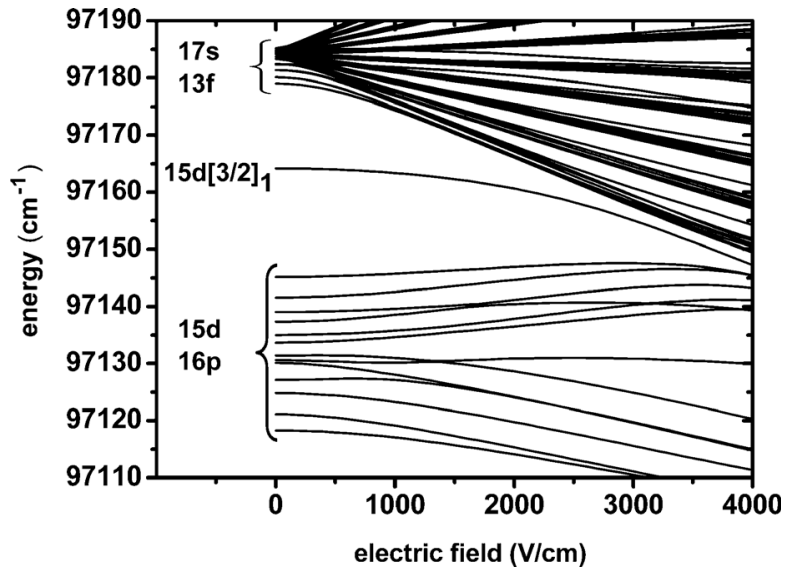

FIG. 6. Stark map for Rydberg energy levels in xenon around the $15 d[3 / 2]_{1}$ state.

our experimental results (Fig. 3), we can determine the energies of the different observed levels and compare them with theoretical Stark maps (Fig. 6). Figure 8 shows that the extra transitions observed were also in agreement with the calculated results. They were caused by mixing between the $15 d[3 / 2]_{1}$ level and levels from the $13 f$ manifold.

\section{FEASIBILITY FOR USE AS ELECTRIC FIELD DIAGNOSTIC}

Following the experimental and theoretical results from the previous sections, we now will discuss the possibility of using Stark shift measurements of Rydberg levels in xenon as a diagnostic for electric fields in low-pressure discharges. We present the procedure to measure electric fields and estimate the accuracy and minimum detectable electric field for our proposed diagnostic. Additionally, possible applications for the diagnostic are identified.

The investigations so far have shown that we can measure Stark shifts of Rydberg levels in xenon atoms. Furthermore, we can also accurately predict these Stark shifts for $n d$ levels

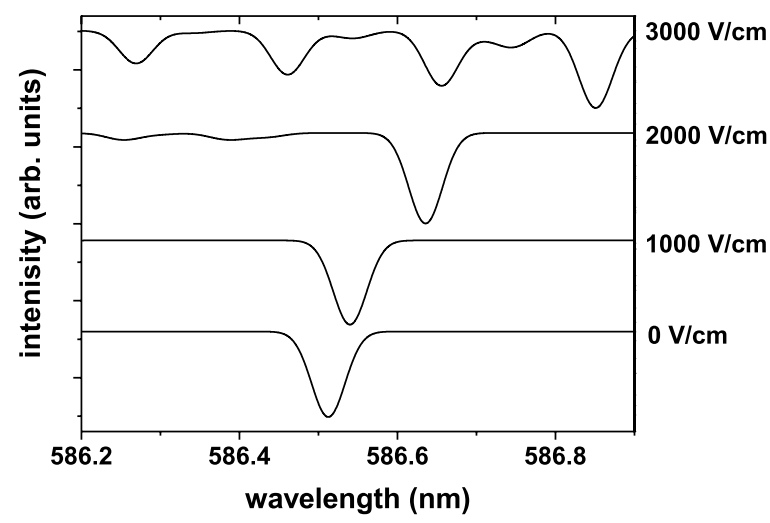

FIG. 7. Theoretical excitation spectrum of the $15 d[3 / 2]_{1}$ state in different electric fields. An offset in the $y$ scale is used to separate the different spectra. The wavelength scale corresponds to the wavelength of the second laser (Fig. 1), exciting from $6 p[1 / 2]_{0}$ to Rydberg states. 


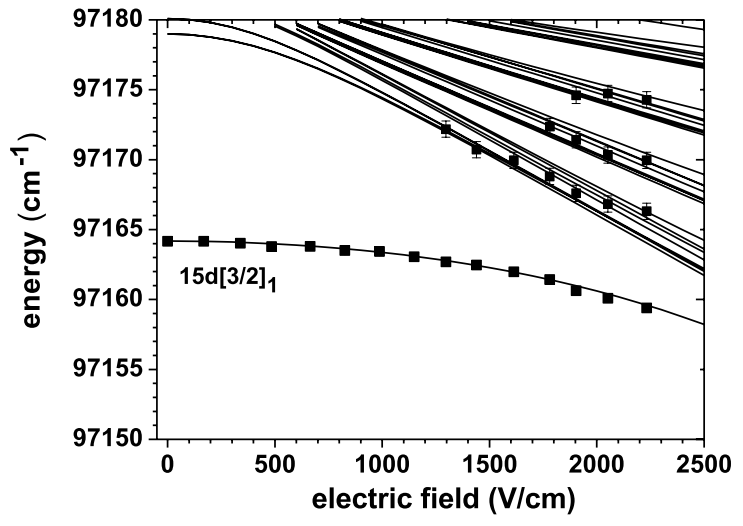

FIG. 8. Theoretical Stark map for energy levels around $15 d[3 / 2]_{1}$ with superimposed experimentally observed transitions. The solid lines are calculations, the squares $(\boldsymbol{\square})$ indicate experimental results.

using a theoretical calculation. Therefore, the nd levels appear to be suitable as an electric field diagnostic by measuring the Stark shifts in unknown electric fields. For the $n s$ levels, the observed shifts were much smaller than for $n d$ levels and the theoretical calculation was less accurate, therefore these levels are less suitable as electric field diagnostic.

Quantification of an unknown electric field strength can be done by matching observed fluorescence-dip spectra with theoretical calculations for different fields. The accuracy of such a diagnostic can be estimated from the comparison between our experiments in known electric fields and the theoretical calculation, shown in Fig. 4. Also the minimum detectable electric field, that is the minimum detectable shift, can be estimated from this figure. Obviously, these specifications are different for the different levels of xenon, because they show different Stark shifts. Table I summarizes the main specifications of the electric field diagnostic using different nd levels.

It should be noted that for $n=12-15$ the maximum electric field was determined by our experimental arrangement, not by limitations in the measurement method. For $n$ $=18$, the maximum field was determined by decreasing signal intensities caused by mixing with neighboring levels.

From Table I it can be concluded that electric fields in the range $250-4000 \mathrm{~V} / \mathrm{cm}$ can be detected with an accuracy of about 50 to $150 \mathrm{~V} / \mathrm{cm}$ by measuring Stark shifts of appropriate levels. For field measurements in low electric fields

TABLE I. Characteristics of using Stark shift measurements of different levels as an electric field diagnostic.

\begin{tabular}{cccc}
\hline \hline & $\begin{array}{l}\text { Minimum } \\
\text { detectable } \\
\text { field }(\mathrm{V} / \mathrm{cm})\end{array}$ & $\begin{array}{l}\text { Measured } \\
\text { maximum } \\
\text { field }(\mathrm{V} / \mathrm{cm})\end{array}$ & $\begin{array}{l}\text { Accuracy } \\
(\mathrm{V} / \mathrm{cm})\end{array}$ \\
\hline $12 d[3 / 2]_{1}$ & 1000 & 4000 & $300-150$ \\
$13 d[3 / 2]_{1}$ & 500 & 2500 & $150-100$ \\
$14 d[3 / 2]_{1}$ & 300 & 2500 & $200-100$ \\
$15 d[3 / 2]_{1}$ & 300 & 2500 & $150-75$ \\
$18 d[3 / 2]_{1}$ & 250 & 1000 & $50-30$ \\
\hline \hline
\end{tabular}

$(250-1000 \mathrm{~V} / \mathrm{cm})$, using the $18 d[3 / 2]_{1}$ level is most suitable. For intermediate fields $(1000-2500 \mathrm{~V} / \mathrm{cm})$, the $15 d[3 / 2]_{1}$ state gives most accurate results. High electric fields $(2500-4000 \mathrm{~V} / \mathrm{cm})$ were only measured using the $12 d[3 / 2]_{1}$ level.

Since we have shown that our theoretical calculation is accurate for calculating Stark shifts for $n=12-15$ and $n$ $=18$, it can be expected that it is also accurate for other similar values of $n$. Therefore, in general for the measurement of high electric fields, levels with low $n$ are most suitable and for detection of low electric fields, the levels with high $n$ are best.

In many low-pressure gas discharges, electric fields of up to a few $\mathrm{kV} / \mathrm{cm}$ can be expected, for instance, sheath regions of glow discharges and moving ionization fronts in plasma breakdown. For these types of discharges, measuring Stark shifts of Rydberg levels is an interesting diagnostic for electric field strengths.

An important advantage of the proposed technique is the use of the ground state of the atom as initial level for excitation. So far, electric field diagnostics for noble gas discharges used metastable atoms as lower level $[15,16,19,20]$. Using the ground state makes it possible to measure electric fields in neutral gases or plasmas with low degrees of ionization, such as ionization fronts in plasma breakdown.

Additionally, by using a LIF detection scheme, electric field distributions in time-varying plasmas, such as $\mathrm{rf}$ discharges, can be monitored. This is not possible using optogalvanic detection methods.

It should be noted that the current technique is limited to discharges containing xenon at relatively low pressure. First, the xenon atoms are needed for detecting Stark shifts. However, xenon may be added to a discharge as a trace gas, extending the possible use of the technique. Additionally, the total gas pressure cannot be too high, because of collisional quenching of the intermediate $6 p[1 / 2]_{0}$ level, which reduces the intensity of the fluorescence signal. Furthermore, pressure broadening will increase the width of the observed dips, making it more difficult to detect small shifts accurately. The maximum pressure for which this diagnostic can be used was not investigated in detail yet, but was estimated to be around $5000 \mathrm{~Pa}$, limited by pressure broadening leading to a dip width of about $6 \mathrm{~cm}^{-1}(200 \mathrm{pm})$ [24].

\section{CONCLUSIONS}

We measured Stark effects in low-pressure xenon gas by LIF-dip spectroscopy. Using a $(2+1)$-photon excitation scheme, starting from the xenon ground state, nd and $n s$ Rydberg levels were investigated in various electric fields. For principal quantum numbers ranging from 12 to 18 , Stark shifts of up to $4.8 \mathrm{~cm}^{-1}$ were measured for electric fields from 0 to $4000 \mathrm{~V} / \mathrm{cm}$.

The Stark effects were also studied using a theoretical calculation. With this relatively simple calculation procedure, we could accurately calculate Stark effects in $n d$ levels. For $n s$ levels, the match with experiments was only moderate. This shows that the main assumption of the calculation, a hydrogenlike atom with nonpenetrating electron orbits, is 
valid for calculating Stark effects of $n d$ Rydberg levels. For $n s$ levels, the deviations were probably caused by deviations from the assumption of a hydrogenlike atom. To accurately calculate Stark effects for these levels, more sophisticated calculation methods are needed.

Measurements of Stark shifts of $n d$ Rydberg levels can be used as an electric field diagnostic in low-pressure gas discharges. With our current technique we can measure electric fields from 250 to $4000 \mathrm{~V} / \mathrm{cm}$ with an accuracy of about 50 to $150 \mathrm{~V} / \mathrm{cm}$. These measurements can be performed with both spatial and temporal resolution. Additionally, the use of the ground state as the lower level in the excitation scheme, makes it possible to measure electric field distributions in neutral gas and weakly ionized plasmas. Furthermore, xenon can be added to a discharge as a trace gas for diagnostic purposes.

A possible application of this diagnostic is the study of electric fields during plasma breakdown. Especially for investigations of the electric field enhancement in moving ionization fronts and the formation process of sheath structures, our proposed technique can be used. Such investigations are currently being performed and first results are expected in the near future.

\section{ACKNOWLEDGMENTS}

The authors wish to thank Uwe Czarnetzki and his group at the Ruhr-Universität Bochum for the useful discussion on LIF-dip spectroscopy. Eddie van Veldhuizen of the Eindhoven University of Technology is acknowledged for his expertise and help with the voltage pulse system. This research was supported by the Nederlandse Organisatie voor Wetenschappelijk Onderzoek (NWO) under the VIDI Innovational Research scheme, Project No. ETF 5856.
[1] M. G. Littman, M. L. Zimmerman, T. W. Ducas, R. R. Freeman, and D. Kleppner, Phys. Rev. Lett. 36, 788 (1976).

[2] R. R. Freeman, N. P. Economou, G. C. Bjorklund, and K. T. Lu, Phys. Rev. Lett. 41, 1463 (1978).

[3] M. L. Zimmerman, M. G. Littman, M. M. Kash, and D. Kleppner, Phys. Rev. A 20, 2251 (1979).

[4] D. E. Kelleher and E. B. Saloman, Phys. Rev. A 35, 3327 (1987).

[5] B. E. Cole, J. W. Cooper, D. L. Ederer, G. Mehlman, and E. B. Saloman, J. Phys. B 13, L175 (1980).

[6] R. D. Knight and L. G. Wang, Phys. Rev. A 32, 896 (1985).

[7] C. Delsart and J. C. Keller, Phys. Rev. A 28, 845 (1983).

[8] P. F. Brevet, M. Pellarin, and J. L. Vialle, Phys. Rev. A 42, 1460 (1990).

[9] C. A. Moore, G. P. Davis, and R. A. Gottscho, Phys. Rev. Lett. 52, 538 (1984).

[10] D. K. Douhty and J. E. Lawler, Appl. Phys. Lett. 45, 611 (1984)

[11] B. N. Ganguly and A. Garscadden, Appl. Phys. Lett. 46, 540 (1985).

[12] J. P. Booth, M. Fadlallah, J. Derouard, and N. Sadeghi, Appl. Phys. Lett. 65, 819 (1994).

[13] J. B. Kim, K. Kawamura, Y. W. Choi, M. D. Bowden, K. Muraoka, and V. Helbig, IEEE Trans. Plasma Sci. 26, 1556
(1998).

[14] U. Czarnetzki, D. Luggenhölscher, and H. F. Döbele, Phys. Rev. Lett. 81, 4592 (1998).

[15] K. Takizawa, K. Sasaki, and K. Kadota, Jpn. J. Appl. Phys., Part 2 41, L1285 (2002).

[16] E. V. Barnat and G. A. Hebner, Appl. Phys. Lett. 85, 3393 (2004).

[17] W. E. Ernst, T. P. Softley, and R. N. Zare, Phys. Rev. A 37, 4172 (1988).

[18] T. Ebata, N. Mikami, and M. Ito, J. Chem. Phys. 78, 1132 (1983).

[19] V. P. Gavrilenko, H. J. Kim, T. Ikutake, J. B. Kim, Y. W. Choi, M. D. Bowden, and K. Muraoka, Phys. Rev. E 62, 7201 (2000).

[20] T. Jiang, M. D. Bowden, E. Wagenaars, E. Stoffels, and G. M. W. Kroesen (unpublished).

[21] A. R. Edmonds, J. Picart, N. Tran Minh, and R. Pullen, J. Phys. B 12, 2781 (1979).

[22] G. Racah, Phys. Rev. 61, 537 (1942).

[23] Y. Ralchenko, F. C. Jou, D. E. Kelleher, A. E. Kramida, A. Musgrove, J. Reader, W. L. Wiese, and K. Olsen, http:// physics.nist.gov/asd3

[24] P. Labastie, E. Giacobino, and F. Biraben, J. Phys. B 15, 2605 (1982). 